\title{
Prospective randomized comparison of open versus laparoscopic management of splenic artery aneurysms: a 10-year study
}

\author{
Guido A. M. Tiberio • Stefano Bonardelli • \\ Federico Gheza $\cdot$ Luca Arru $\cdot$ Edoardo Cervi • \\ Stefano M. Giulini
}

Received: 9 December 2011 / Accepted: 21 May 2012

(C) The Author(s) 2012. This article is published with open access at Springerlink.com

\begin{abstract}
Background The literature does not support the choice between open and laparoscopic management of splenic artery aneurysms (SAA).

Methods We designed a prospective, randomized comparison between open and laparoscopic surgery for SAA. Primary end points were types of surgical procedures performed and clinical outcomes. Analysis was developed on an intention-to-treat basis.

Results Fourteen patients were allocated to laparotomy (group A) and 15 to laparoscopy (group B). Groups displayed similar patient- and aneurysm-related characteristics. The conversion rate to open surgery was $13.3 \%$. The type of surgical procedure performed on the splenic artery was similar in the two groups: aneurysmectomy with splenic artery ligature or direct anastomosis was performed in $51 \%$ and $21 \%$ of patients in group A and in $60 \%$ and $20 \%$ in group B, respectively. The splenectomy rate was similar (14\% vs. $20 \%)$. Postoperative splenic infarction was observed in one case in each group. Laparoscopy was
\end{abstract}

Registration Number: NCT01387828.

G. A. M. Tiberio - F. Gheza - L. Arru - S. M. Giulini General Surgery, Department of Medical and Surgical Sciences, University of Brescia, Brescia, Italy

G. A. M. Tiberio $(\bowtie)$

c/o Clinica Chirurgica-III, Divisione di Chirurgia Generale, Spedali Civili di Brecia1, Piazzale Spedali Civili,

25100 Brescia, Italy

e-mail: guido.tiberio@libero.it

S. Bonardelli · E. Cervi

Vascular Surgery, Department of Medical and Surgical Sciences,

University of Brescia, Brescia, Italy associated with shorter procedures $(p=0.0003)$ and lower morbidity ( $25 \%$ vs. $64 \%, p=0.045$ ). Major morbidity requiring interventional procedures and blood transfusion was observed only in group A. Laparoscopy was associated with quicker resumption of oral diet $(p<0.001)$, earlier drain removal $(p=0.046)$, and shorter hospital stay $(p<0.01)$. During a mean follow-up of 50 months, two patients in group A required hospital readmission. In group $\mathrm{B}$, two patients developed a late thrombosis of arterial anastomoses.

Conclusions Our study demonstrates that laparoscopy permits multiple technical options, does not increase the splenectomy rate, and reduces postoperative complications. It confirms the supposed clinical benefits of laparoscopy when ablative procedures are required but laparoscopic anastomoses show poor long-term results.

Keywords Clinical paper $\cdot$ Trials $\cdot$ Splenic artery aneurysms

Recent literature reports on the feasibility, safety, and effectiveness of laparoscopic management of splenic artery aneurysms (SAA) as well as its appreciation by patientsoften young females-who harbour the disease [1-13]. Thus, laparoscopy may represent an interesting alternative to open surgery and provided that the same range of technical solutions offered by open surgery can be achieved with similar results $[1,14-18]$ and the general advantages of laparoscopy are confirmed in this specific application, the laparoscopic option may represent a challenging alternative not only to laparotomic surgery but also to endovascular procedures.

Endovascular management, albeit minimally invasive, requires accurate patient selection and presents some 
critical points: recanalization (in up to $12.5 \%$ of cases), coil migration and distal infarction with abscess formation, and, rarely, aneurysm rupture have all been described, often in association with the so-called postembolization syndrome (fever \pm ileus \pm pain \pm pancreatitis in up to $30 \%$ of cases) after transcatheter embolization. Endovascular stent grafts are indicated for proximally located aneurysms but long-term results remain unknown [18]. For these reasons, at our institution endovascular treatment is indicated in emergency situations or for critical patients not fit for surgery, while surgery is considered the best solution for patients requiring elective management of SAA.

This study reports the first prospective randomized comparison of the different surgical techniques for SAA management, and it is one of the few randomized studies that compares open and laparoscopic surgery.

\section{Patients and methods}

Between January 2001 and December 2010 all patients requiring elective management of SAA were offered the opportunity to enter a prospective randomized open-label comparison study between open and laparoscopic surgery. The study, performed on an intention-to-treat basis, was approved by the Institutional Ethics Committee.

In all cases, preoperative work-up included a multidetector angio-CT. Indications for surgery were an aneurysm diameter $\geq 2 \mathrm{~cm}$ or $<2 \mathrm{~cm}$ if in presence of diameter increase, saccular shape or child-bearing age, according to prevailing literature $[1,5,6,18-21]$, if the main trunk was involved. In the case of a hilar branches' aneurysm, in absence of clear guidelines, surgery was indicated for lesions exceeding $250 \%$ of the caliber of the involved branch. Patients with an aneurysm involving the celiac trunk and those corrected during synchronous major open procedures were excluded. Previous abdominal surgery was not a contraindication for laparoscopic surgery.

Patients received complete information about the data from the literature, all alternative treatments, and the results of our own series of patients who had already undergone open or laparoscopic treatment of SAA. Regardless of group assignment, patients were operated on by the surgeon most experienced in open and laparoscopic techniques in order to eliminate biases linked to a surgeon's technical preference.

Surgical technique

A supine position was adopted for both open and laparoscopic procedures and the table was rotated as necessary. Laparotomic surgery was conducted through a subcostal incision. For the laparoscopic approach, a $30^{\circ}$ optic was introduced 3-5 $\mathrm{cm}$ above the umbilicus and three or four trocars were introduced along a hypothetical bilateral subcostal incision. The surgeon was positioned between the patient's legs in the case of proximal lesions and at the patient's right side in the case of distal aneurysms. In all cases the incision of the lesser sac respected the short gastric vessels. Proximal control of the splenic artery invariably preceded aneurysmectomy. Aneurysm dissection along a periadventitial plane generally was achieved by monopolar or bipolar electrocautery, but LigaSure ${ }^{\mathrm{TM}}$ forceps (Covidien, Mansfield, MA, USA) were also employed, especially during laparoscopy.

Arterial reconstruction was performed only if there was main trunk involvement, whenever the arterial wall appeared normal, and the gap after aneurysmectomy allowed a direct end-to-end anastomosis. Carrel's triangulation technique was adopted in open anastomoses, and a single running suture of $5-0$ or $6-0$ prolene or PTFE was used in laparoscopic procedures. The technical result was verified by intraoperative continuous wave Doppler ultrasound.

Splenectomy was indicated for aneurysms deeply immersed in the splenic hilum or for massive parenchymal ischemia after artery ligation. For segmental severe ischemia, partial splenectomy concluded the procedure. An aspiration drain was routinely left in place. Postoperative medical treatment included a prophylactic vaccine schedule whenever splenectomy was performed; in this case chronic antiplatelet medication was also indicated.

Variables considered in the study

The comparison of the two surgical approaches was performed on the basis of patient-related, aneurysm-related, operative, and postoperative variables, with the latter including both short- and long-term results. Patient-related variables included demographic data, BMI, and ASA score; aneurysm-related variables included aneurysm location, number, shape, and size (of the largest if there were multiple aneurysms). Operative variables included conversion to open surgery; type of procedure performed on the splenic artery and its branches; associated procedures on the spleen; incidence and extent of splenic ischemia, defined as relative (change of color and consistency in the presence of arterial signal at Doppler exploration) or severe (dark and soft parenchyma, no Doppler signal); duration of surgery; blood loss; and transfusion needs. If synchrnous procedures were performed, operative time and blood loss were those of the SAA treatment alone. Early postoperative results considered surgical and medical morbidity (Clavien-Dindo classification [22]), analgesic needs, time to oral intake, time to drain removal, and length of hospital stay. After discharge, patients were submitted to a clinical 
control 30 days after surgery. A second control was scheduled after 6 months; this included an angio-CT or angio-MRI in those cases of splenic artery reconstruction and an ultrasound evaluation of the spleen and its vascularization in the other cases. Laboratory evaluations were performed 9-12 months after surgery in order to investigate splenic function using platelet and lymphocyte count and peripheral blood smear. The follow-up schedule included an ultrasound 1 year after surgery and telephone interviews thereafter. Long-term results were matched with data obtained from the regional computer registry of hospital activities in order to evaluate all postoperative hospital admissions.

Randomization and statistics

Given the small number of cases, we scheduled a permuted-block randomization [23], with an allocation ratio of $1: 1$ but with a random block size. Block dimension was defined by generating a series of random pairs between 6 and 40 . As a result, a stochastic binary (A and B) sequence of 36 elements was generated in order to contain the same number of As and Bs. The random series was known only by the referent of randomization process. We programmed to close the study in 10 years and to generate a number of random binary blocks sufficient to include all enrolled patients.

Data are expressed as mean or median \pm standard deviation or range: statistical analysis was performed by Student's $t$ test and Fisher's exact test for qualitative data using SPSS 16.0® (IBM Corporation, Somers, NY, USA); $p<0.05$ was rated as significant.

The low incidence of the disease did not allow statistical power concern, however, the G*Power 3.1.2 software provided by Düsseldorf University (free software, Erdfelder, Faul, and Buchner, 1996) permitted a post hoc evaluation of the statistical power of some of our results with a predetermined $\alpha$ error of 0.05 . It was possible to investigate: duration of surgery, time to oral intake, time to drain removal and length of hospital stay.

\section{Results}

The proposed trial was accepted by all but one patient who decided to postpone the intervention. Twenty-nine patients (22 females), ranging in age from 33 to 76 years, were randomized between open (group A, 14 patients) and laparoscopic (group B, 15 patients) management of SAA (Fig. 1). All SAAs were clinically silent, and eight of them were discovered while investigating nonspecific abdominal symptoms. In group B, three patients underwent simultaneous procedures: cholecystectomy in one case,

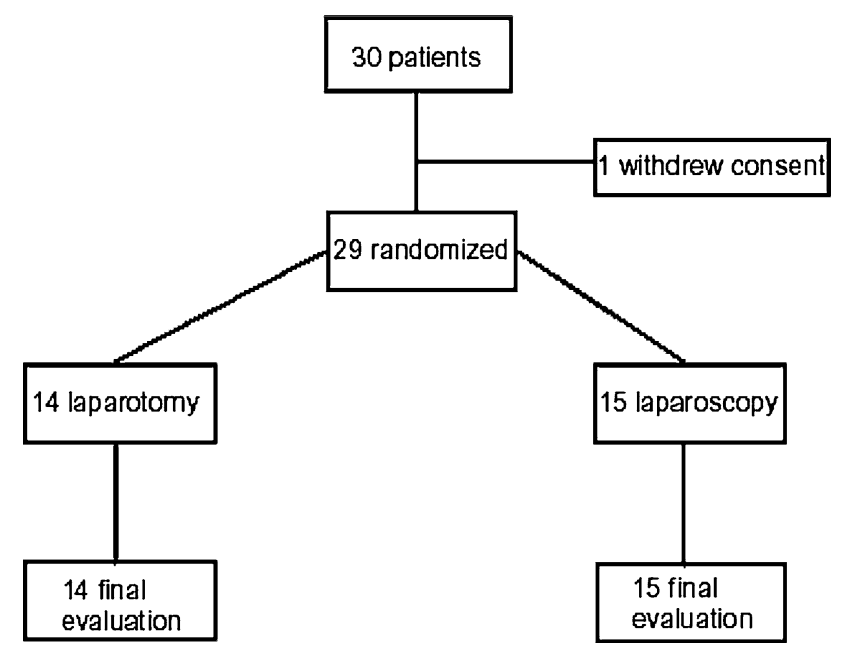

Fig. 1 Trial profile

Table 1 Patient and aneurysm characteristics

\begin{tabular}{llll}
\hline & $\begin{array}{l}\text { Group A } \\
\text { (open surgery) } \\
(n=14)\end{array}$ & $\begin{array}{l}\text { Group B } \\
(\text { laparoscopy })\end{array}$ & $p$ \\
$(n=15)$ & \\
\hline Patient characteristics & & & \\
Age & 60 & 59 & n.s. \\
Sex ratio F:M & $10: 4$ & $12: 3$ & n.s. \\
BMI (mean \pm SD) & $25.5 \pm 2.8$ & $26.5 \pm 2.3$ & n.s. \\
ASA (mean) & 2.3 & 2.2 & n.s. \\
Aneurysm characteristics & & & \\
Single & 10 & 9 & n.s. \\
$\begin{array}{l}\text { Double } \\
\text { Multiple (>2) }\end{array}$ & 3 & 3 & n.s. \\
$\begin{array}{l}\text { Saccular } \\
\text { Fusiform }\end{array}$ & 6 & 3 & n.s. \\
$\varnothing$ median (range) & $28(15-50)$ & $25.5(15-50)$ & n.s. \\
$\quad$ (mm) & & 8 & n.s. \\
Main trunk & $8(57 \%)$ & $6(40 \%)$ & n.s. \\
Bifurcation/hilar & $6(43 \%)$ & $9(60 \%)$ & n.s. \\
branch/es & & & \\
\hline
\end{tabular}

n.s. not significant

endovascular exclusion of abdominal aortic aneurysm in the second, and epigastric hernia repair in a third. The two groups had similar patient-related and aneurysm-related characteristics (Table 1).

Operative and postoperative mortality was nil. The conversion rate from laparoscopic to open surgery was $13.3 \%$ (two cases). One conversion case was due to aneurysm penetration into the pancreatic tail which was treated by splenopancreatectomy, and the other conversion case was due to a tear in a venous branch during dissection of a hilar aneurysm which was repaired by suturing. 
Table 2 Surgical procedures and operative results
${ }^{a}$ Including one conversion to open surgery for hemostasis

${ }^{\mathrm{b}}$ Including one conversion to open surgery for splenectomy and pancreatectomy

\begin{tabular}{llll}
\hline & $\begin{array}{l}\text { Group A } \\
\text { (open surgery) } \\
(n=14)\end{array}$ & $\begin{array}{l}\text { Group B } \\
\text { (laparoscopy) } \\
(n=15)\end{array}$ & $p$ \\
\hline $\begin{array}{l}\text { Surgical procedures } \\
\text { Aneurysmectomy + artery ligation }\end{array}$ & $7(51 \%)$ & $9(60 \%)^{\mathrm{a}}$ & n.s. \\
$\begin{array}{l}\text { Aneurysmectomy + end-to-end anastomosis } \\
\text { Aneurysmectomy + splenectomy }\end{array}$ & $3(21 \%)$ & $3(20 \%)$ & n.s. \\
Aneurysmectomy + partial splenectomy & $2(14 \%)$ & $3(20 \%)^{\mathrm{b}}$ & n.s. \\
Operative results & $2(14 \%)$ & 0 & 0.0129 \\
Conversion to open surgery & & $2(13.3 \%)$ & \\
Partial splenic ischemia & $2 / 10(20 \%)$ & $7 / 12(58.3 \%)$ & 0.004 \\
Blood loss & Negligible & Negligible & n.s. \\
Operative time (min) (median \pm SD) & $190 \pm 48$ & $125 \pm 34$ & 0.0003 \\
\hline
\end{tabular}

The type of surgical procedure performed on the splenic artery was similar in the two study groups (Table 2). In particular, after aneurysmectomy, splenic artery ligation or end-to-end anastomosis was performed in $51 \%$ and $21 \%$ of patients in group A and in $60 \%$ and $20 \%$ in group B, respectively. Arterial reconstruction was not dictated by splenic ischemia, which was never observed in the case of lesions affecting the main trunk of the splenic artery, but was performed in order to restore normal circulation. At declamping, intraoperative continuous wave Doppler was always normal.

After arterial ligation for distally located lesions, the spleen was managed conservatively by means of resection of a severely ischemic caudal pole in two open-surgery patients (14\%); this condition was not encountered in the laparoscopic patients. On the other hand, the splenectomy rate was similar in the two groups (14\% vs. $20 \%)$. For distally located lesions, adequate control of the splenic hilum always required the interruption of the left gastroepiploic artery during open surgery, but in laparoscopic procedures it was possible to preserve the same artery because it was easy to achieve an adequate working space by gentle elevation of the gastrosplenic ligament (Fig. 2).

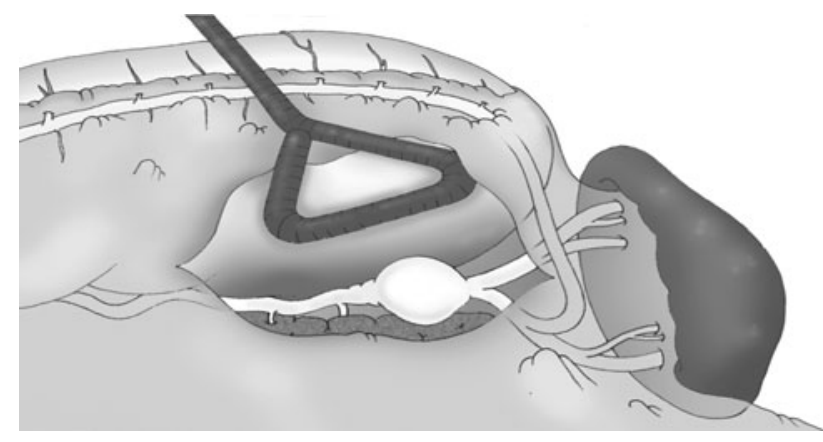

Fig. 2 Laparoscopy allows easy preservation of the left gastroepiploic artery
When the spleen was not resected, the rate of relative ischemia of a portion of the splenic parenchyma was $20 \%$ and $58.3 \%(p=0.004)$ in groups $\mathrm{A}$ and $\mathrm{B}$, respectively (Table 2). Postoperative imaging controls showed an effective revascularization of the ischemic areas in all but two cases, one in each group, in which a segmental splenic infarction with progressive uncomplicated fibrous retraction was documented by serial US scans. Both cases had the gastroepiploic artery ligated, the patient in group B during laparotomic conversion for hemostasis.

In general, operative blood loss was negligible.

Laparoscopy allowed for shorter procedures ( $p=$ 0.0003; statistical power $98 \%$ ), quicker resumption of oral diet $(p=0.0014$; statistical power $93 \%)$, earlier drain removal $(p=0.046$; statistical power $50 \%)$, and shorter hospital stay ( $p=0.010$; statistical power $74 \%$ ). Quantity, quality, and duration of pain medication were similar in both groups (Table 3). Laparoscopy was also associated with a lower overall morbidity rate (57\% vs. $21 \%, p=0.045)$, as seen in Table 3. In particular, grade III morbidity requiring interventional procedures was observed only in group A: one patient developed early postoperative hemorrhagic shock requiring surgical hemostasis, and another patient, who had a partial splenectomy, developed abdominal collections requiring percutaneous drainage. Accordingly, transfusions were limited to group A as these two patients received blood transfusions. Minor postoperative morbidity was equally represented in the two groups. At a mean follow-up of 50 months, two patients (group A) required hospital readmission: one was readmitted three times for subocclusive attacks that were always managed nonoperatively, and one developed an incisional hernia requiring reintervention.

The proposed radiologic control of vascular reconstruction was accepted by four of six patients, two in each group. It showed patent anastomoses after open surgery and thrombosis with collateral perfusion of the spleen after laparoscopic surgery. 
Table 3 Postoperative results

\begin{tabular}{llll}
\hline & $\begin{array}{l}\text { Group A } \\
\text { (open surgery) } \\
(n=14)\end{array}$ & $\begin{array}{l}\text { Group B } \\
\text { (laparoscopy) } \\
(n=15)\end{array}$ & $p$ \\
\hline Analgesia (days) (median \pm SD) & $2 \pm 1.6$ & $2 \pm 1.2$ & 0.078 \\
Oral diet (days) (median \pm SD) & $4 \pm 1.3$ & $2 \pm 1.5$ & 0,001 \\
Drain removal (days) (median \pm SD) & $5,5 \pm 2.8$ & $4 \pm 1.2$ & 0.046 \\
Hospital stay (days) (median \pm SD) & $8 \pm 2.8$ & $6 \pm 1.8$ & 0.010 \\
Postoperative major morbidity (grade III) & & 0 & \\
$\quad$ Hemorrhagic shock & 1 & 0 & \\
$\quad$ Intra-abdominal collection & 1 & 0 & \\
Postoperative minor morbidity (grade I-II) & & 2 & \\
$\quad$ Mild pancreatitis & 1 & 1 & \\
Respiratory complications & 2 & 0 & \\
$\quad$ Splenic infarction & 1 & 0 & \\
Long-term postoperative morbidity & & $20 \%$ & \\
Intestinal subocclusion & 1 & & \\
Incisional hernia & 1 & $57 \%$ & \\
Total morbidity & & & \\
\hline
\end{tabular}

Table 4 Postoperative splenic function

\begin{tabular}{llllll}
\hline & $\begin{array}{l}\text { Normal } \\
\text { spleen }(n=8)\end{array}$ & $\begin{array}{l}\text { Partial splenic } \\
\text { ischemia }(n=5)\end{array}$ & $\begin{array}{l}\text { Partial splenic } \\
\text { infarction }(n=2)\end{array}$ & $\begin{array}{l}\text { Partial } \\
\text { splenectomy }(n=2)\end{array}$ & $\begin{array}{l}\text { Splenectomy } \\
(n=5)\end{array}$ \\
\hline Platelets $\times 1,000 /$ cc (median) & 239 & 221 & 210 & 330 & 424 \\
Lymphocytes (\%) & 27.3 & 34 & 27.5 & 41 & 39.6 \\
Anisocytosis & $0 \%$ & $0 \%$ & $0 \%$ & $0 \%$ & $100 \%$ \\
Poikilocytosis & $0 \%$ & $0 \%$ & $0 \%$ & $0 \%$ & $100 \%$ \\
\hline
\end{tabular}

Postoperative control of splenic function was obtained in 22/29 patients. Normal parameters were seen in all cases of spleen preservation, including two cases of partial splenic infarction and five cases of partial splenic ischemia. Patients who underwent partial splenectomy showed thrombocytosis and lymphocytosis, associated with anisocytosis and poikilocytosis after splenectomy (Table 4).

\section{Discussion}

This is the first prospective randomized comparison of open and laparoscopic surgery for treating aneurysmal disease of the splenic artery, using a small but unselected and homogeneous group of patients. In general terms our data show the clinical advantages of less invasive surgery that were already described for other applications of the laparoscopic technique and highlight some points that deserve comment.

First, it should be noted that laparoscopy was not restricted to simple ablative surgery, limited to the aneurysm or extended to the spleen, and that the different technical options were indifferently ruled out with the open and the laparoscopic technique, with the exception of partial splenectomy which was performed only in open surgery but was never indicated during laparoscopic procedures. In particular, we observed a similar incidence of ablative and reconstructive arterial procedures in the two groups, showing that unusual laparoscopic procedures like arterial reconstruction by end-to-end anastomosis can be performed when the main branch of the splenic artery is concerned.

Laparoscopic vascular anastomosis in the splanchnic area has been reported mainly on the splenic artery, which favors the procedure because of its easy-to-reach position, safe vessel control, and by the fact that a procedure fault leading to thrombosis has low risk of severe consequences due to the efficient collateral circulation in this area. In fact, despite the normal intraoperative continuous wave Doppler US, we observed late thrombosis of two laparoscopic anastomoses, both uncomplicated. The literature (summarized in Table 5) does not provide insight into long-term results of 
Table 5 Experiences from literature addressing laparoscopic approach to SAA through the analysis of more than three cases

\begin{tabular}{|c|c|c|c|c|c|c|}
\hline & Arca & Reardon & Obuchi & Pietrabissa & Giulianotti & This work \\
\hline Patient number & 4 & 6 & 6 & 16 & 9 & 15 \\
\hline Mean operative time (min) & 150 & 187 & 177 & 143 & 212 & 125 \\
\hline Conversion rate $(\%)$ & 17 & 20 & - & 0 & 0 & 13.3 \\
\hline Vascular anastomoses & & & & $1^{\mathrm{a}}$ & $6^{\mathrm{b}}$ & $3^{c}$ \\
\hline Splenectomy rate $(\%)$ & 50 & - & 67 & 19 & 11 & 20 \\
\hline Partial splenectomy (\%) & - & - & - & - & 11 & 0 \\
\hline Morbidity (\%) & - & - & 17 & - & - & 20 \\
\hline Oral diet (days) & 1,5 & 1 & 2 & - & 1,7 & 2 \\
\hline Drain removal (days) & - & - & 3 & - & - & 4 \\
\hline Hospital stay (days) & - & - & 8 & - & 7,1 & 6 \\
\hline
\end{tabular}

${ }^{\text {a }}$ Robotic

b Robotic, two cases with graft interposition

${ }^{c}$ Laparoscopic

laparoscopic reconstructions $[1,3,24]$; we speculate that a technical defect linked to the running suture may have played a role in this negative evolution. Lack of stability of the surgical field caused by excessive movement of the arterial stumps in our experience favored the running suture technique, which was adopted also to speed up the procedure. In the future we shall replicate Carrel's triangulation which was adopted in the open procedures, introducing, if necessary, more graspers in order to stabilize the surgical field. From this point of view we must consider the advantages of robotic surgery in terms of surgical effectiveness [24]. In light of the uneventful thrombosis of our anastomoses, we must also reconsider the role of vascular reconstruction itself if collateral circulation is preserved, keeping in mind that in surgery one should seek to obtain the targeted result without unnecessary additional procedures.

Our results confirm the observation of Pietrabissa et al. [3] that distally located aneurysms are more difficult to manage and entail a higher risk of associated splenectomy. However, we found that the laparoscopic approach also was effective for treating lesions involving splenic artery bifurcation and/or its hilar branches, managed by aneurysmectomy and arterial ligation with full respect for collateral circulation, left gastroepiploic artery included. The similar rate of splenic infarction in the two study groups despite a higher incidence of hilar lesions in the laparoscopic group should be ascribed to the preservation of an efficient collateral vascularization, allowing adequate arterial supply to the caudal segment of the spleen through the left gastroepiploic artery. We never observed splenic infarction if splenic collateral circulation was sustained not only by short gastric vessels (routinely preserved) but also by the left gastroepiploic artery. The latter can be easily preserved during laparoscopic procedures by gentle elevation of the gastrosplenic ligament which allows for easy control of the splenic hilum, particularly if a $30^{\circ}$ scope is employed (Fig. 2).

In light of the efficient blood flow redistribution shown by postoperative US imaging, intraoperative detection of a slight change of color and consistency of a portion of the splenic parenchyma, here defined as "relative ischemia," should be considered a transient modification.

Splenic preservation is of paramount importance in these patients, considering their young age and that risks of asplenic status are lifelong [25-28]. In our experience, intraoperative Doppler ultrasonography proved invaluable in supporting a conservative approach whenever arterial blood flow, albeit demodulated, was detected by the probe placed on the spleen's surface; more sound information can be achieved by duplex or echo-color-Doppler devices [3]. The uneventful evolution of the observed segmental infarctions does not support the need for systematic resection of ischemic splenic parenchyma. It is a complex procedure and associated with specific morbidity (one case of partial splenectomy developed postoperative collections requiring percutaneous drainage and blood transfusion), the indication of which must be accurately weighted in light of a risk/ benefit balance. The normality of splenic function at late controls in case of splenic infarction further supports a conservative attitude.

In our experience an aneurysm deeply penetrating into the pancreatic tail and splenic hilum was managed by splenopancreatectomy; we must here emphasize that in the case of a difficult dissection of the aneurysm, an alternative and less invasive option is represented by proximal and distal vessel ligation followed by aneurysm fenestration without aneurysmectomy and control of bleeding from collateral vessels by simple suture.

Laparoscopic management of SAA of the main trunk of the splenic artery is not difficult, but distal lesions and 
reconstructive procedures can require solid laparoscopic skills. In our experience, the collaboration of vascular and general surgeons was fruitful and offered a greater chance for improvement for those concerned, with appreciable dropout in term of results and benefits for our patients. In particular, it promoted advanced laparoscopy in a discipline that is at present more oriented to the development of endovascular procedures than to the evolution of surgical techniques.

At our institution surgery is still considered the best solution for patients requiring elective management of SAA because of the lack of strong data on the long-term results of endovascular treatments. However, we must stress that we are aware of the role of endovascular surgery, especially if it is able to duplicate surgical procedures by means of stent grafting or vascular occlusion; endovascular management must always be considered among the preeminent therapeutic options, particularly if the most recent generation of stents can be used.

In conclusion, our study, limited by the small number of patients but with the strength of a prospective randomized trial, demonstrates the supposed clinical benefits of laparoscopy when ablative procedures are required and describes a work in progress as far as laparoscopic anastomoses are concerned. It also shows that laparoscopy permits multiple technical options in order to adapt the procedure to the individual case, does not increase the splenectomy rate even in case of distal and hilar aneurysms, and reduces postoperative complications.

Disclosure Drs. Guido A. M. Tiberio, Stefano Bonardelli, Federico Gheza, Luca Arru, Edoardo Cervi, and Stefano Maria Giulini have no conflicts of interest or financial ties to disclose.

Open Access This article is distributed under the terms of the Creative Commons Attribution License which permits any use, distribution, and reproduction in any medium, provided the original author(s) and the source are credited.

\section{References}

1. Ha JF, Sieunarine K (2009) Laparoscopic splenic artery aneurysm resection: review of current trends in management. Surg Laparosc Endosc Percutan Tech 19:e67-e70

2. Arca M, Gagner M, Heniford B et al (1999) Splenic artery aneurysms: methods of laparoscopic repair. J Vasc Surg 30:184-188

3. Pietrabissa A, Ferrari M, Berchiolli R et al (2009) Laparoscopic treatment of splenic artery aneurysms. J Vasc Surg 50:275-279

4. Suzuki H, Shimura T, Asao T et al (2002) Laparoscopic resection of splenic artery aneurysm; a case report. Hepatogastroenterology 49:1520-1522

5. Obuchi T, Sasaki A, Nakajima J et al (2009) Laparoscopic surgery for splenic artery aneurysm. Surg Laparosc Endosc Percutan Tech 19:338-340
6. Reardon PR, Otah E, Craig ES et al (2005) Laparoscopic resection of splenic artery aneurysms. Surg Endosc 19:488-493

7. De Csepel J, Quinn T, Gagner M (2001) Laparoscopic exclusion of a splenic artery aneurysm using a lateral approach permits preservation of the spleen. Surg Laparosc Endosc Percutan Tech 11:221-224

8. Sandford RM, Lloyd DM, Ross Naylor A (2006) Laparoscopic ligation of splenic artery aneurysm. Surg Laparosc Endosc Percutan Tech 16:102-103

9. Adham M, Blanc P, Douek P et al (2000) Laparoscopic resection of a proximal splenic artery aneurysm. Surg Endosc 14:372

10. Meinke AK, Floch NR, Dicorato MP (2002) Laparoscopic options in the treatment of splenic artery aneurysms. Surg Endosc 16:1107

11. Mastracci T, Cadeddu M, Colopinto R, Cinà C (2005) A minimally invasive approach to the treatment of aberrant splenic artery aneurysms: a report of two cases. J Vasc Surg 41:1053-1057

12. Holdsworth R, Gunn A (1992) Ruptured splenic artery aneurysm in pregnancy. A review. Br J Obstet Gynaecol 99:595-597

13. Ha JF, Phillips M, Faulkner K (2009) Splenic artery aneurysm rupture in pregnancy. Eur J Obstet Gynecol Reprod Biol 146:133-137

14. Abbas MA, Stone WM, Fowl RJ et al (2002) Splenic artery aneurysms: two decades experience at Mayo Clinic. Ann Vasc Surg 16:442-449

15. Jung SI, Joh YG, Um JW, Suh SO (2001) The Seoul experience of splenic artery aneurysms. Ann Chir Gynaecol 90:10-14

16. Pulli R, Innocenti A, Barbanti E et al (2001) Early and long-term results of surgical treatment of splenic artery aneurysms. Am J Surg 182:520-523

17. Kokkalera U, Bhende S, Ghellai A (2006) Laparoscopic management of splenic artery aneurysms. J Laparoendosc Adv Surg Tech 16:604-608

18. Al-Habbal Y, Christophi C, Muralidharan V (2010) Aneurysms of the splenic artery - A review. Surgeon 8:223-231

19. Heestand G, Sher L, Lightfoote J et al (2003) Characteristics and management of splenic artery aneurysm in liver transplant candidates and recipients. Am Surg 69:933-940

20. Mattar SG, Lumsden AB (1995) The management of splenic artery aneurysms: experience with 23 cases. Am J Surg 169:580-584

21. Moon DB, Lee SG, Hwang S et al (2009) Characteristics and management of splenic artery aneurysms in adult living donor liver transplant recipients. Liver Transpl 15:1535-1541

22. Dindo D, Demartines N, Clavien PA (2004) Classification of surgical complications: a new proposal with evaluation in a cohort of 6336 patients and results of a survey. Ann Surg 240:205-213

23. Schulz KF, Grimes DA (2002) Generation of allocation sequences in randomized trials: chance, not choice. Lancet 359:515-519

24. Giulianotti PC, Buchs NC, Coratti A et al (2011) Robot-assisted treatment of splenic artery aneurysms. Ann Vasc Surg 25:377-383

25. Lee SY, Florica O (2008) Laparoscopic resection of splenic artery aneurysm with preservation of splenic function. Singapore Med J 49:303-304

26. Jones P, Leder K, Woolley I et al (2010) Postsplenectomy infection - strategies for prevention in general practice. Aust Fam Physician 39:383-386

27. Spelman D, Buttery J, Daley A et al (2008) Guidelines for the prevention of sepsis in asplenic and hyposplenic patients. Intern Med J 38:349-356

28. Eichner ER (1979) Splenic function: normal, too much and too little. Am J Med 66:311-320 